\title{
Subjective Evaluation of Cooling Fan Sound based on Grade Scoring and Paired Comparison
}

\author{
Lifang Yang ${ }^{1, a}$, Rui Zhu ${ }^{1, b}$ \\ ${ }^{1} 2054$ School of Mechanical Engineering Building, 92 West Dazhi St., Harbin, P.R.China

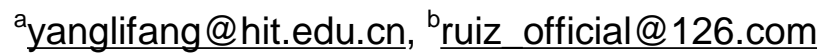

Keywords: Subjective evaluation, Correlation coefficient, Cooling fan sound quality

Abstract. As an essential equipment, the fan is widely used in industrial and civil fields for gas compression and gas delivery. Though the cooling fan can keep us comfortable temperature, it bring the annoying noise. Usually noise reduction will first be conducted to the cooling fan, however, the sound quality of cooling fan is also an important factor to annoy the people. In the paper, sound quality evaluation model for cooling fans will be established to evaluate the sound. It helps screen the objective parameters, including loudness, sharpness, etc. Also, it serves as the output when training the Back Propagation (BP) Neural Networks. Thus, it is important to find an appropriate method to complete the subjective evaluation experiment. In this test, various subjective evaluation methods are compared. And the best method for cooling fan sound quality evaluation is selected by analyzing the correlation coefficient between subjective and objective evaluation result.

\section{Introduction}

Cooling fans are almost applied everywhere as the industry booming. It is placed in the machine room to keep indoor temperature normal and machine running well. As shown in Fig.1, the cooling fan for industry use is always placed in the community and residents is bothered by the noise of it. What's more, the noise of it is not constant, which means people have to get used to the sound repeatedly every time it produces noise.

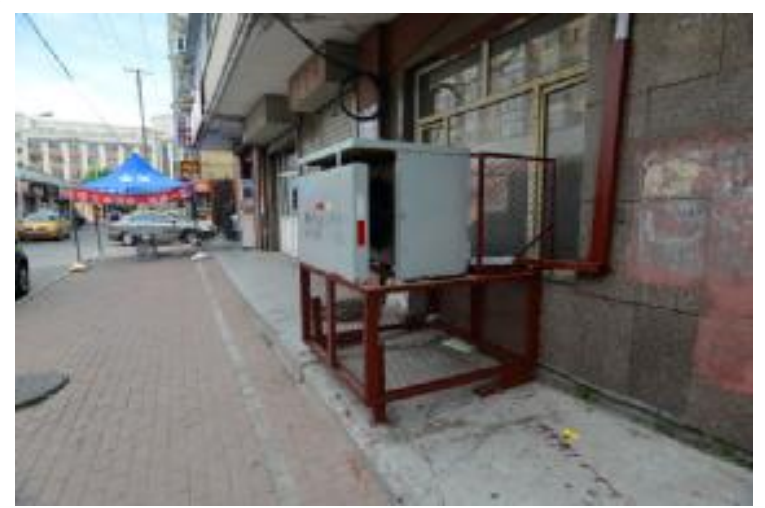

Figure 1. Cooling fan placed in the community.

Yet, these useful cooling fans also cause lots of noise. The noise more than $60 \mathrm{~dB}(\mathrm{~A})$ will annoy the workers. If the noise keep getting strong and up to $75 \mathrm{~dB}(\mathrm{~A})$, it will influence the work efficiency and harm people's hearing system [1]. It is too late and meaningless to find problems of noise after it did harm to human hearing system. Nowadays, noise deduction has been made to reduce the harmful noise. The result indicates that the sound level becomes lower but people in community still feel annoyed. And completely reduce the noise is not practical, also takes high cost. So the subjective evaluation is important for noise reduction. Subjective evaluation is an essential part in establishment of evaluation model, working as the dependent variable in the nonlinear regression model and training model of BP Neural Network as input. There are plenty of subjective evaluation methods, such as, Grade Scoring Method, Paired Comparison Method, Semantic Differential Method, Numerical Estimation Method, etc.

Semantic Differential Method describe samples with an adjective and its antonym, for example, noisy and quiet, weak and strong. Between the adjective and antonym, there usually are 5 to 7 levels. And the levels are divided by adverbs, such as, very noisy, really quiet, etc. [2]. Numerical Estimation 
Method as a simple method, it is a method that ask subjects grade on each sample. This method does not need to train subjects beforehand. But subjects without experience will grade rather discretely. Thus, it is a method which suits Expert Users better [3]. Grade Scoring Method and Paired Comparison Method will be used and introduced in the following experiment.

There are also other methods, but the listed ones are the most commonly used. The subjective evaluation result should express the subjective feeling of a majority of users, so the methods have to suit the users without experience and not trained. Thus, subjective experiments are designed based on Grade Scoring Method and Paired Comparison Method which are easy to understand.

\section{Collection of Sound Samples and Selection of Test Subjects}

Collecting and Editing Cooling Fan Sound Samples. The collection of sound samples happened in a signal station in Heilongjiang Province. There are both TV and radio signal transmissions and different types of cooling fans are used in these two sections. Sound samples were collected on the spots including right and left, front and back, the furthest and closest working spots, etc., as shown in Fig.2 (a).

13 samples with duration of 180s were collected with the HMS IV produced in HEAD Acoustic. As shown in Fig.2 (b), the wave of sound can be monitored in the collecting process and make corrections when errors happen. The meaningless parts in sound samples were cut in the editing process. 33 sound samples with duration of $5 \mathrm{~s}$ were formed when edit was done.

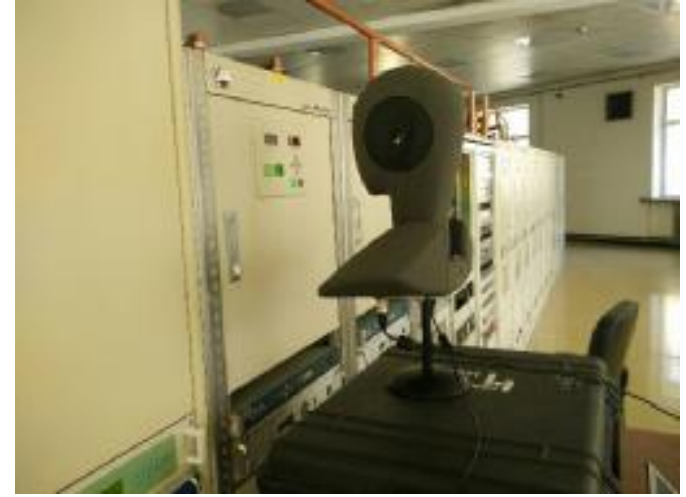

(a)

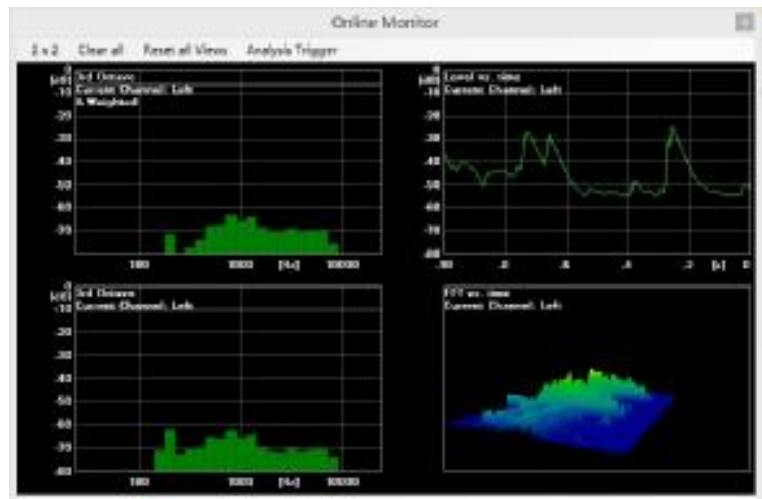

(b)

Figure 2. Online monitor of sound waves.

Test Subjects Selection. The subjective experiment recruited 60 subjects. 30 subjects out of them are for the Grade Scoring test and the rest are for the Paired Comparison. The ratio of gender is nearly 1:1. And all of them possess normal hearing.

\section{Subjective Experiment based on Grade Scoring Method}

Grade Scoring. In Grade Scoring Method, the subjects will be asked to mark the levels of all test samples on one standard. And each level owns a grade. After grading all the test samples, arithmetic average of each test sample will be calculated [4]. For instance, there is a sample named $A$ and 10 subjects grading sample $A$ with marks of $x_{1}, x_{2}, \ldots, x_{\mathbf{1 0}}$, So the mark of the sample $A$ is as follows:

$$
\mathrm{M}_{\mathrm{A}}-\frac{\mathrm{x}_{1}-\mathrm{x}_{2}+\ldots+\mathrm{x}_{10}}{10}
$$

And the result $\mathrm{M}_{\mathrm{A}}$ is the mark of each test sample. This method is easy to understand and operate. But for those subjects not experienced, it will be hard to mark accurately for the first time.

Experiment of Grade Scoring. Number of testing samples is 33 in total. And each is named with number, in case subjects will be affected by the names. As shown in Fig.3, the experimenter played each sound sample to the subjects during experiment. And subjects should grade the sample with mark of $0,0.2,0.4,0.6,0.8,1.0$. The mark of 1.0 means the noisiest sound while " 0 " is for the quietest sample. 


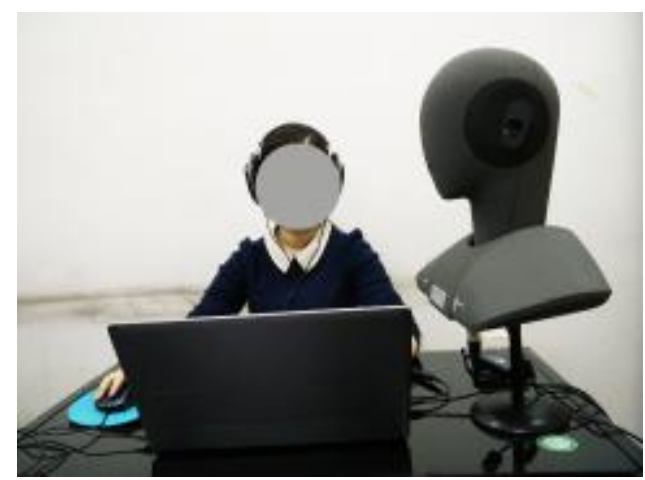

Figure 3. Subjective experiment scene of Grade Scoring Method.

Playing sound samples to subjects will cost 165 s. There will be a 60 -second break when every 11 samples are finished playing in case subjects will feel tired. 30 subjects graded 33 testing samples with the method of Grade Scoring. Marks of each sample were calculated as shown in Table 1, according to the Eq. 1.

Table 1 Marks of sound samples based on Grade Scoring

\begin{tabular}{cccccccccc}
\hline No. & Mark & No. & Mark & No. & Mark & No. & Mark & No. & Mark \\
\hline 1 & 0.03 & 8 & 0.356 & 15 & 0.195 & 22 & 0.582 & 29 & 0.79 \\
2 & 0.51 & 9 & 0.561 & 16 & 0.368 & 23 & 0.693 & 30 & 0.62 \\
3 & 0.042 & 10 & 0.408 & 17 & 0.432 & 24 & 0.862 & 31 & 0.462 \\
4 & 0.241 & 11 & 0.214 & 18 & 0.625 & 25 & 0.368 & 32 & 0.772 \\
5 & 0.301 & 12 & 0.203 & 19 & 0.501 & 26 & 0.896 & 33 & 0.68 \\
6 & 0.191 & 13 & 0.132 & 20 & 0.852 & 27 & 0.632 & & \\
7 & 0.409 & 14 & 0.452 & 21 & 0.592 & 28 & 0.982 & & \\
\hline
\end{tabular}

\section{Subjective Experiment based on Paired Comparison Method}

Paired Comparison. Before grading with Paired Comparison Method, all the samples have to be paired. Then subjects will be asked to compare and choose one of two samples based on some standard [5]. Name two samples in a pair $i$ and $j, \mathrm{P}_{\mathrm{ij}}$ is the probability that subjects choose sample $i$, while $\mathrm{P}_{\mathrm{ij}}$ is the probability of selecting sample $j$. If the number of samples is $t$ in total, then the mark of sample $i$ is as follows:

$$
M_{i}=\frac{1}{t} \sum_{i \tau j} \ln \left(\frac{p_{i j}}{p_{i i}}\right)
$$

Experiment of Paired Comparison. 33 samples were paired into 528 pairs. Under ideal condition, the experiment will last 2640s, i.e. about $90 \mathrm{~min}$. In case subjects feeling tired, every half an hour, they will be asked to take a rest. Experimenter played sound samples in ArtemiS to subjects. One sample out of a pair named $A$, the other is named $B$. Subjects were invited to grade the pair according to irritability degree. There are marks of $0,0.2,0.4,0.6,0.8,1$. Marks of 33 samples are as shown in Table 2 according to Eq. 2 after testing.

Table 2 Marks of sound samples based on Pair Comparison

\begin{tabular}{cccccccccc}
\hline No. & Mark & No. & Mark & No. & Mark & No. & Mark & No. & Mark \\
\hline 1 & -1.126 & 8 & -0.162 & 15 & -0.524 & 22 & 0.412 & 29 & 1.350 \\
2 & -1.090 & 9 & -0.251 & 16 & 0.205 & 23 & 0.440 & 30 & 1.253 \\
3 & -1.147 & 10 & -0.207 & 17 & 0.245 & 24 & 0.423 & 31 & 0.794 \\
4 & -0.719 & 11 & -0.225 & 18 & 0.264 & 25 & 0.087 & 32 & 0.792 \\
5 & -0.648 & 12 & -0.305 & 19 & -0.106 & 26 & 0.090 & 33 & 0.823 \\
6 & -0.712 & 13 & -0.549 & 20 & -0.082 & 27 & 0.055 & & \\
7 & -0.164 & 14 & -0.521 & 21 & -0.113 & 28 & 1.317 & & \\
\hline
\end{tabular}

Correlation Analysis between Objective and Subjective Parameters of Cooling Fan Sound Samples 
Objective Evaluation of 33 Sound Sample. There are three types of parameters for the objective evaluation, including psychoacoustic parameters, sound pressure level and A-weighed sound level. Psychoacoustics indicates how brain interprets the sound human beings hear [6]. Here in the paper, loudness, sharpness, fluctuation strength, roughness, tonality, speech intelligibility and articulation index will be analyzed with ArtemiS. Sound pressure level describes whether sound is noisy or not [7]. However, it cannot show how people feel about the sound.

Compared with sound pressure level, B-weighted and C-weighted sound levels, A-weighted sound level is closer to the human feeling [8]. Imported 33 samples, the objective parameters are received.

Correlation Analysis on Grade Scoring Marks and Objective Parameters. Importing objective parameters as well as subjective evaluation results based on Grade Scoring, carried on correlation analysis in SPSS. The result of correlation analysis is as shown in Table 3.

Table 3 Correlation Coefficient regarding to Grade Scoring

\begin{tabular}{cccc}
\hline Objective Parameters & Coefficient & Objective Parameters & Coefficient \\
\hline Sharpness & $0.779^{* *}$ & Articulation Index & $-0.714^{* *}$ \\
Loudness & $0.783^{* *}$ & Speech Intelligibility & $-0.626^{* *}$ \\
Fluctuation Strength & $0.810^{* *}$ & Sound Pressure Level & $0.727^{* *}$ \\
Roughness & $0.788^{* *}$ & A-weighted Sound Level & $0.752^{* *}$ \\
Tonality & $-0.365^{* *}$ & & \\
\hline
\end{tabular}

Fluctuation strength is highly relevant to the subjective evaluation based on Grade Scoring with coefficient of 0.810. Sharpness, loudness, roughness, articulation index, speech intelligibility, sound pressure level and A-weighted sound level are remarkably relevant to the subjective evaluation. And tonality is in low correlation with subjective marks.

Besides, tonality, articulation index and speech intelligibility are in negative correlation with subjective parameters while others are in positive correlation.

Correlation Analysis on Paired Comparison Marks and Objective Parameters. Input all the objective data and Paired Comparison results into SPSS and calculate the correlation coefficient. The analysis result is as shown in Table 4.

Table 4 Correlation Coefficient regarding to Paired Comparison

\begin{tabular}{cccc}
\hline Objective Parameters & Coefficient & Objective Parameters & Coefficient \\
\hline Sharpness & $0.914^{* *}$ & Articulation Index & $-0.781^{* *}$ \\
Loudness & $0.956^{* *}$ & Speech Intelligibility & $-0.674^{* *}$ \\
Fluctuation Strength & $0.902^{* *}$ & Sound Pressure Level & $0.857^{* *}$ \\
Roughness & $0.899^{* *}$ & A-weighted Sound Level & $0.847^{* *}$ \\
Tonality & $-0.493^{* *}$ & & \\
\hline
\end{tabular}

Sharpness, loudness, fluctuation strength, roughness, sound pressure level and A-weighted sound level are in a high correlation with subjective evaluation of Paired Comparison. Articulation index and speech intelligibility are remarkably relevant to subjective marks. And tonality is in lower correlation with subjective evaluation.

3 objective parameters including tonality, articulation index and speech intelligibility are in negative relation with subjective evaluation results based on Paired Comparison, while others are in positive correlation.

Comparison between Grade Scoring and Paired Comparison. Correlation coefficient of Grade Scoring and Paired Comparison methods and objective evaluation result is as shown in Fig.4. All the correlation coefficients are in form of absolute value. 


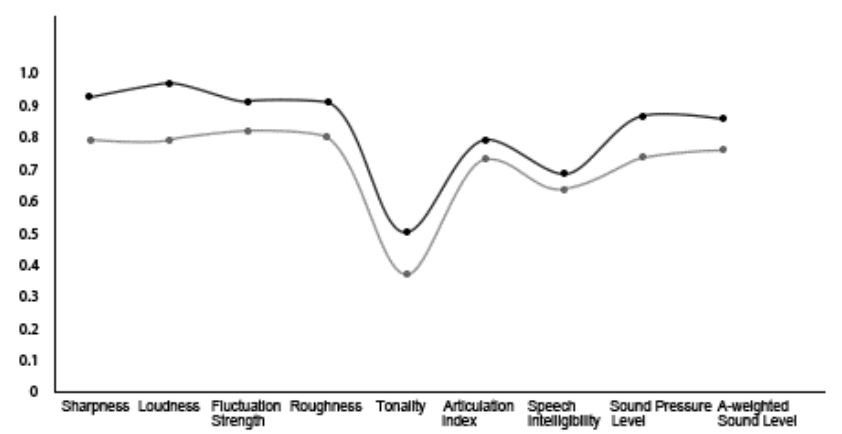

Figure 4. Comparison between Grade Scoring and Paired Comparison marks.

The top black line stands the correlation coefficient of Paired Comparison Method, while the bottom grey line is for the correlation coefficient of Grade Scoring Method. It is obvious that the correlation of Paired Comparison with objective parameters is much higher than Grade Scoring. So while establishing evaluation model, Paired Comparison Method is a better choice for subjective experiment. Because of the higher correlation coefficient, Paired Comparison makes subject and objective evaluation more relevant to each other.

\section{Conclusions}

To conclude, Paired Comparison is a better choice for subjective evaluation as it possesses a higher correlation coefficient with objective parameter. Tonality has the lowest correlation coefficient of 0.493 , which means tonality is in a low correlation with subjective parameters. So in the following establishment of evaluation model, tonality will be removed while other parameters are saved. Most of parameters are in positive correlation with subjective evaluation and only tonality, articulation index and speech intelligibility are negatively relevant.

From the conclusions, the Grade Scoring method is better for the samples with even difference. And the differences of them can be distinguished as level 1, level 2, etc. But for those samples not possessing uniformed difference, the Paired Comparison method is a better choice. Because the comparison is between each pair but not one compared with the rest.

Thus, in this situation that Irritability Degree of sound samples is not evenly distributed, the Paired Comparison method is well suited.

\section{References}

[1] Z. Y. Zhang: Control and measures on urban noise pollution, The Merchandise and Quality Vol. 10 (2011), p. 220

[2] Chouard N, Hmpel T: A semantic differential design especially developed for the evaluation of interior car sounds, Berlin, Germany (1999)

[3] J. Liang: Research on analysis and evaluation method of vehicle interior sound quality based on binaural auditory model, Changchun, PRC (2007)

[4] Q. Sun: Research on evaluation and application of vehicle sound quality based on artificial neural network, Changchun, PRC (2010)

[5] Parizet E: Paired comparison listening tests and circular error rates, Acta Acustica united with Acustic Vol. 88 (2002), p. 594-598

[6] Zwicker E, Fastl H: Psychoacoustics, facts and models, Berlin, Germany (1999)

[7] H. M. Ding: Research on objective evaluation of air condition sound quality and software development, Nanjing, PRC (2002)

[8] M. D. Zhang: The weakness of A-weighted sound level in measurement and evaluation on noise, Technical Acoustics Vol. 01 (1991), p. 41-42. 